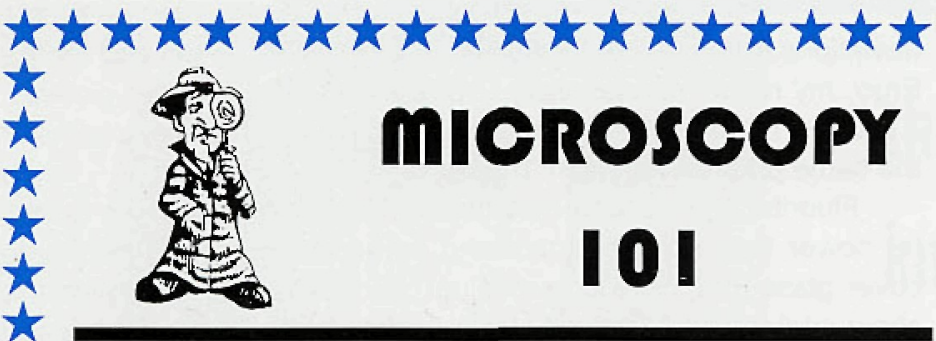

We appreciate the response to this publication feature - and welcome all contributions. Contributions may be sent to Phil Oshel, our Technical Editor at:
Mr. Phil Oshel
(608)833-2885
PO Box $620068 \quad$ Fax: (608)836-1969
Middleton WI 53562 eMail: oshel@terracom.net

\section{Stereo Zoom}

Ask any optical microcopist if their zoom stereo microscope stays in focus throughout the full range of its zoom and the chances are that they will say "no". They will accept, as a design fault, the need to readjust the scope as they go through the zoom range. The problem, however, does not lie with the microscope design but with the microscopist who has not correctly adjusted the scope. To set up your zoom stereomicroscope so that it remains in focus throughout its full range, here is what to do:

Set the individual eyepiece diopter adjustments to approximately the middle of their range (forget what your spectacle prescription says!)

At the highest zoom range, adjust the microscope focus control for sharpest image.

Zoom to the lowest end of the magnification range.

Bring the object back into focus for each eye using the eyepiece dopter adjustment ONLY. DO NOT REFOCUS WITH THE FOCUS KNOB.

Zoom back up to the highest magnification and check that the object is still in focus, fine tune with the focus control if necessary. Your scope now remains in focus over the entire zoom range.

\section{Ian Stewart}

\section{Coating Coverslips So Cells Adhere}

Coverslips coated with laminin, collagen, fibronectin or other matrix materials are useful for growing adherent cells, as the cells often do not adhere well to glass alone. To make our own coverslips for fluorescent confocal microscopy work, we use the following protocol. The matrix material used, either alone or in combination, varies depending on cell type. For most breast cancer cell lines we use a combination of laminin and collagen IV. B16BL6 or CHO cells stick well to many matrix materials.

1) Preclean good quality glass coverslips with soap, rinse with water, rinse with acetone, rinse with distilled water several times, then air dry.

2) Expose them to UV light to sterilize.

3) Transfer the coverslips to an appropriately sized sterile petri dish.

4) Place several drops (6 or 7 ) of $50 \mu \mathrm{g} / \mathrm{mL}$ matrix material onto the clean dry coverslips and spread gently with a Pasteur pipette. Let air dry overnight.
For cell cultures:

Transfer a coverslip to an individual petri dish, gently place a drop or two of cells in suspension on the coverslips and dilute dropwise with an appropriate amount of serum-containing medium to spread the cells across the coverslip. After allowing the cells to adhere for a few hours, add more medium and incubate until cells are ready for use.

Deborah L. Berglund, Montana State University

\section{An SEM Preparation for Spray Dried Highly Hygroscopic Magnesium Chloride}

Obviously, $\mathrm{MgCl}_{2}$ specimens are stored and opened in a dry nitrogen environment, such as a glove box.

1) Take a clean graphite SEM mount, previously stored in desiccator.

2) Draw a thin film of Apiezon vacuum grease over the mount (using a glass slide).

3) Open the $\mathrm{MgCl}_{2}$ specimen container, dip the greased SEM mount surface into the salt.

4) Quickly sputter coat with gold, and observe immediately at low kV.

The method worked every time I used it and preparation times were in minutes. There was no particulate contamination of SEM optics, charging was minimized by low kV operation, particle size distribution was easily achieved, and EDS analysis was possible.

\section{William G. Schultz, Schlumberger ATE}

\section{How To Dry Reagents Using Molecular Sieves}

Molecular sieves are a family of adsorbents that can be used to dry gases and organic liquids. They have a crystalline structure and come in different types depending on the size of the pores in the crystals. For example, type $3 \mathrm{~A}$ is named for the size of the pores ( 3 Angstroms), and is used to remove moisture from unsaturated hydrocarbons and polar fluids. These would include methanol, ethanol, and acetone. Type $3 \mathrm{~A}$ will adsorb a molecule less than $3 \AA$ in size (e.g., water) and has an adsorption capacity of $22 \%$ by weight.

To dry polar liquids such as methanol, add a slight excess of drying agent to the liquid. The water specification (this can be found in the manufacturer's catalog or on a certificate of analysis) may state that the water content is $1.0 \%$. This equates to $40 \mathrm{~mL}$ in a 4 liter bottle. $40 \mathrm{~mL}$ of water is equal to $40 \mathrm{~g}$ of water. $40 \mathrm{~g}$ divided by $22 \%=181.8 \mathrm{~g}$. To insure an excess, use $200 \mathrm{~g}$ of molecular sieve. Keep in mind that an opened bottle of methanol, which is very hygroscopic, would probably have a higher moisture content, depending on how many times the bottle has been opened for use. How often you would need to dry a solvent is dependent on your application, use, and humidity.

Add the determined amount of molecular sieve (for example, EM Science part number MX1583D/1), mix thoroughly and allow the liquid to stand. After a few minutes, the drying agent settles to the bottom of the container. Separation can be completed by decanting or filtration (suction filtration would work best and fastest). After use, the molecular sieve can be regenerated (dried) by heating a thin layer in an oven at $350^{\circ}$ to $500^{\circ} \mathrm{F}\left(\sim 180^{\circ}\right.$ to $260^{\circ}$ C) for about an hour. It is then once again ready for use.

Rande Kline and Joe Daniels, EM Science 